\title{
What Does The Korringa Ratio Measure?
}

\author{
B. Sriram Shastry \\ AT \& T Bell Laboratories, Murray Hill, New Jersey 07974 \\ Elihu Abrahams \\ Serin Physics Laboratory, Rutgers University, PO Box 849, Piscataway, New Jersey 08855
}

(24 June 1993)

\begin{abstract}
We present an analysis of the Korringa ratio in a dirty metal, emphasizing the case where a Stoner enhancement of the uniform susceptibilty is present. We find that the relaxation rates are significantly enhanced by disorder, and that the inverse problem of determining the bare density of states from a study of the change of the Knight shift and relaxation rates with some parameter, such as pressure, has rather constrained solutions, with the disorder playing an important role. Some preliminary applications to the case of chemical substitution in the $\mathrm{Rb}_{3-x} \mathrm{~K}_{x} \mathrm{C}_{60}$ family of superconductors is presented and some other relevant systems are mentioned.
\end{abstract}

\section{INTRODUCTION}

Nuclear magnetic resonance (NMR) is an important experimental tool for elucidating the physics of electronic materials whose properties are determined by strong electron-electron correlations. For example, NMR data for high- $T_{c}$ superconductors and for low dimensional conductors has given us important information about their electronic properties. Furthermore, such data are key benchmarks against which theoretical approaches must be measured.

Basic NMR measurements in a metal involve the magnetic resonance field shift $K$ and the spin relaxation rate $1 / T_{1}$ of a given nucleus in the material. [1] The effects of electron-electron interactions on these quantities have been studied theoretically for nearly forty years 2.4 and it is often taken for granted that a deviation of the "Korringa ratio" $\mathcal{K}=1 /\left(T_{1} T K^{2}\right)$ from a constant is a signature of the effect of strong correlations. In particular, the standard canon is that if the Korringa ratio exceeds unity, it is evidence for an antiferromagnetic enhancement of the electron susceptibility away from zero wave vector which enters $1 / T_{1}$, [4] while if $\mathcal{K}$ is less than unity it is because there is a (ferromagnetic) Stoner enhancement of the uniform susceptibility which enters $K$. [2, 3] It is our purpose to point out that the well-known corrections to the susceptibility due to disorder [5] influence the Korringa ratio in an important way which must be considered when drawing conclusions from the experimental results. We shall show that disorder significantly enhances the Korringa ratio and that it must be included when an attempt is made to estimate Stoner factors from $\mathcal{K}$. In what follows, we shall not consider the case of antiferromagnetic enhancements of the wave-vector dependent electron susceptibility. Our discussion of the ferromagnetic Stoner enhancement is sufficient to illustrate the point.

When the resonance field shift is determined solely by the local spin polarization of the conduction electrons, it 
is called the Knight shift and it is proportional to the hyperfine coupling $g \gamma_{N} A$ and the uniform conduction electron susceptibility $\chi(\mathbf{q} \rightarrow 0, \omega=0)$. Here, $\gamma_{N}$ is the nuclear gyromagnetic ratio so that $A$ has dimension of magnetic field. At the same time, $1 / T_{1}$ depends on $A^{2}$ and on a density of electron magnetic excitations which is given by the imaginary part of $\chi$. The latter is averaged over all wave vectors weighted by a form factor [6] which depends on the position of the nucleus in the unit cell and may also include the effect of a transferred hyperfine interaction. Thus we have

$$
\begin{gathered}
K=A \chi(0,0) / \mu_{B} \\
1 / T_{1}=k_{B} T\left(\gamma / \mu_{B}\right)^{2} \sum_{\mathbf{q}} A^{2}(\mathbf{q}) \chi^{\prime \prime}\left(\mathbf{q}, \omega_{0}\right) / \omega_{0},
\end{gathered}
$$

where $A(\mathbf{q})$ is the "q-dependent hyperfine" coupling which involves the form factor mentioned above.

The case of free electrons is particularly simple. The susceptibility is of the Lindhard form:

$\chi_{L}^{\prime}(q, \omega)=\chi_{P}\left[1-\left(q / 2 k_{F}\right)^{2} / 3\right], \quad \chi_{L}^{\prime \prime}(q, \omega)=\left(\pi \chi_{P} / 2\right)\left(\omega / v_{F} q\right) \Theta\left(v_{F} q-\omega\right)$,

to leading order in $q, \omega$. Here $\chi_{P}=m^{*} k_{F} \mu_{B}^{2} / \pi^{2}=$ $2 \rho_{0} \mu_{B}^{2}$ is the Pauli susceptibility, $\rho_{0}$ the one-spin density of states at the Fermi level. With one atom per unit cell, the form factor is unity and Eqs. $(1,2)$ combine to give the well-known Korringa relation:

$$
1 / T_{1} T=\pi k_{B}\left(\gamma_{N} / \mu_{B}\right)^{2} K^{2} / 4
$$

which makes it appear that $1 / T_{1}$ is proportional to the square of the uniform susceptibility, or to the square of the density of states. Conclusions have often been drawn from this. However, this dependence is accidental; from Eq. (2) we see that $1 / T_{1} \propto \chi / \Gamma$, where $\Gamma$ is a characteristic energy associated with the susceptibility at the important range of wave vectors in the sum. In any case, the Korringa product will cease to be temperature independent whenever the susceptibility is temperature dependent.

In what follows, we show that diffusive corrections to $\chi(\mathbf{q}, \omega)$ which occur in a disordered metal [5] can also enhance $1 / T_{1}$ and must be considered when the mean free path is short, as is the case, for example, in some high- $T_{c}$ samples and certainly in $\mathrm{M}_{3} \mathrm{C}_{60}$. []]

\section{THE CALCULATION}

We start with the usual RPA result for the susceptibility $\chi(q, \omega)$

$$
\chi(q, \omega)^{-1}=\chi_{0}(q, \omega)^{-1}-U / 2,
$$

where $U$ is the effective Coulomb interaction and $\chi_{0}$ is the dynamical susceptibility, incorporating the effects of 
disorder, but not those of interactions. We take units such that $\hbar=\mu_{B}=1$. We can express $\chi_{0}$ as a sum over impurity lines, renormalizing propagators and vertices to leading order in the impurity scattering rate. This gives rise to the usual diffusion propagator [8] for small $q, \omega$. We can express the result in the form

$$
\chi_{0}(q, \omega) \simeq \chi_{L}(q, \omega) \frac{D_{0} q^{2}}{D_{0} q^{2}-i \omega},
$$

where $\chi_{L}$ is the Lindhard susceptibility for the undisordered, non-interacting system. $D_{0}=\frac{1}{3} v_{F} \lambda$ is the diffusion constant with $\lambda$ the mean free path. To lowest order in disorder and interaction, the mean free path is the same one as enters the resistivity (for point impurities). However, in general, the spin-triplet electron-hole channel mean free path enters the susceptibility, and it can be quite different (smaller) from the one involved in charge transport in the interacting system [5]. We have constructed Eq. (6) so that it has the correct behavior for both large and small $q$. Thus, it is correct to leading order in the disorder, in the limit of low frequencies, and to $O\left(q^{2}\right)$ in the $q$ dependence. We note the leading behaviour for small $\omega$

$$
\chi_{0}^{\prime \prime}(q, \omega) / \omega=\rho_{0}\left(\frac{\pi}{2 q v_{F}}+\frac{1}{D_{0} q^{2}}\right),
$$

This expression is valid for essentially all the range $q<$ $2 k_{F}$, and shows that the Landau damping [the first term in Eq. (7)] ceases to dominate when the dimensionless number $\pi q \lambda / 6<1$. Then the diffusion mechanism dominates the damping. Also to lowest order, the real part of $\chi$ is unchanged by disorder and is still given by Eq. (3).

In order to calculate the NMR relaxation rate, we need $\chi^{\prime \prime}\left(q, \omega_{0}\right) / \omega_{0}$ which is obtained from Eq. (5) as

$$
\frac{\chi^{\prime \prime}\left(q, \omega_{0}\right)}{\omega_{0}}=\frac{\chi_{0}^{\prime \prime}\left(q, \omega_{0}\right)}{\omega_{0}} \frac{1}{\left[1-U \chi_{0}^{\prime}(q, 0) / 2\right]^{2}} .
$$

In Eq. (8), we neglected a term $\left[U \chi_{0}^{\prime \prime}\left(q, \omega_{0}\right)\right]^{2}$ in the denominator since at the low NMR frequency $\omega_{0}$, it vanishes as $\omega_{0}^{2}$. The Stoner denominator in Eq. (8) is sensitive to $\chi_{0}^{\prime}(q, 0)$. Instead of using the approximation of Eq. (6) for it, we can find the result which is valid for all q as follows:

At $\omega=0$ there are no diffuson (i.e. vertex) corrections to $\chi_{0}^{\prime}(q, \omega)$ and it is given simply by

$$
\chi_{0}(\mathbf{q}, 0)=-2 \sum_{k} \frac{n(\mathbf{k}+\mathbf{q})-\mathbf{n}(\mathbf{k})}{\epsilon_{\mathbf{k}+\mathbf{q}}-\epsilon_{\mathbf{k}}} .
$$

When the impurity scattering rate $\Gamma=v_{F} / 2 \lambda$ is larger than the temperature (we take $\hbar=1$ ),

$$
n(\mathbf{k})=\frac{1}{2}-\frac{1}{\pi} \arctan \frac{\epsilon_{\mathbf{k}}}{\Gamma}
$$

where the kinetic energy $\epsilon_{\mathbf{k}}$ is measured from the chemical potential. 
We perform the angular integrals in Eq. (9) in two and three dimensions and find

$$
\begin{aligned}
& \chi_{0}^{\prime}(q, 0)=2 \frac{\rho_{0}}{t} \int_{0}^{t} x d x \frac{n\left(x k_{F}\right)}{\sqrt{t^{2}-x^{2}}} \\
& \chi_{0}^{\prime}(q, 0)=\frac{\rho_{0}}{t} \int_{0}^{\infty} x d x n\left(x k_{F}\right) \ln \left|\frac{t+x}{t-x}\right|,
\end{aligned}
$$

where $x=k / k_{F}$ and $t=q / 2 k_{F}, \eta$ is $k_{F} \lambda$, and $\rho_{0}$ is the one-spin Fermi surface density of states in the appropriate dimension. These may be evaluated in closed form as $q \rightarrow 0$, where we find $\chi^{\prime}(0,0)=2 \rho_{0} n(\mathbf{k}=0)$ in $2 \mathrm{D}$ and $\chi^{\prime}(0,0)=2 \rho_{0} \sqrt{1+\sqrt{\left(1+\eta^{-2}\right)}} / \sqrt{2}$ in $3 \mathrm{D}$. These imply that disorder changes the effective density of states at the Fermi level by a small amount given by these formulae, and for consistency, we must use these factors in estimating the enhancement of the susceptibility or Knight shift.

The result for the NMR relaxation rate is obtained by using Eqs. $(7,11,12)$ in Eq. (8) and integrating over $q$. The results are conveniently expressed in terms of the free electron result:

$$
1 / T_{1} T=\pi k_{B}\left(\gamma_{N} \rho_{0} A / 2\right)^{2} \cdot \mathcal{S}\left(\rho_{0} U, k_{F} \lambda\right),
$$

where the enhancement factor $\mathcal{S}\left(\rho_{0} U, k_{F} \lambda\right)$ contains all the effects of interactions and disorder. In terms of the reduced variables, we have

$$
\begin{aligned}
& \mathcal{S}\left(\rho_{0} U, \eta\right)=\int_{\kappa}^{1} d t \frac{1+(2 / \pi \eta t)}{\left[1-U \chi_{0}^{\prime}(t, 0)\right]^{2}} \\
& \mathcal{S}\left(\rho_{0} U, \eta\right)=2 \int_{0}^{1} t d t \frac{1+(3 / \pi \eta t)}{\left[1-U \chi_{0}^{\prime}(t, 0)\right]^{2}}
\end{aligned}
$$

In Eq. (15), we have introduced the infra-red cutoff $\kappa$ which is determined by an inelastic scattering ("Thouless") length beyond which the diffusion ceases. In particular, $\kappa \simeq 1 / \sqrt{\eta k_{F} \lambda_{i}}$, where $\lambda_{i}$ is the inelastic mean free path. These integrals can be evaluated for $U=0$, where we find $\mathcal{S}\left(\rho_{0} U, \eta\right)=1+6 /(\pi \eta)$ in $3 D$ and in $2 D$, $\mathcal{S}\left(\rho_{0} U, \eta\right)=1+\log \left(k_{F}^{2} \lambda \lambda_{i}\right) /(\pi \eta)$. Note that $\mathcal{S}$ is in general bigger than unity due simply to the enhanced density of states at low energies implied by the diffusive character of spin fluctuations in a disordered metal.

A leading approximation for $\mathcal{S}$ is obtained from Eqs. $(14,15)$ by replacing $\chi_{0}^{\prime}(t, 0)$ by, in $3 D$, the approximate form $2 \rho_{0}\left(1-t^{2} / 3\right)$ of Eq. (3). In $2 D$, the same approximation is $\chi_{0}^{\prime}(t, 0) \simeq 2 \rho_{0}$. Therefore, the approximate expressions for the enhancement factors are

$$
\begin{aligned}
& \mathcal{S}\left(\rho_{0} U, \eta\right)=\frac{1}{\left(1-U \rho_{0}\right)^{2}}\left[1-\frac{2 \ln \kappa}{\pi \eta}\right] \\
& \mathcal{S}\left(\rho_{0} U, \eta\right)=\frac{1}{\left(1-U \rho_{0}\right)^{2}}\left\{\frac{1}{1+y^{2}}+\frac{3}{\pi k_{F} \lambda}\left[\frac{1}{1+y^{2}}+\frac{1}{y} \arctan y\right]\right\},
\end{aligned}
$$

where $y=\sqrt{U \rho_{0} /\left[3\left(1-U \rho_{0}\right)\right]}$. 
We can also calculate the dimensionless Korringa ratio $\mathcal{K}$ (essentially the ratio of $1 / T_{1} T$ to the square of the Knight shift $K$ ) as

$$
\mathcal{K}=\mathcal{S}\left(\chi_{0} / \chi\right)^{2}
$$

This number is also a measure of the interactions and disorder present in a Fermi system, and is of course merely an alternative description to that implied by $\mathcal{S}$. However, in some systems such as $\mathrm{M}_{3} \mathrm{C}_{60}$, the Knight shift is not easy to estimate, since the measured shift has to be apportioned into the chemical and Knight shifts with large uncertainties in both, whence we prefer to present both $\mathcal{K}$ and $\mathcal{S}$.

\section{CONCLUSIONS}

We show some figures which illustrate the discussion of this paper. In Fig. 1, we show how disorder affects the enhancement factor $\mathcal{S}$ in three dimensions. Even when the interaction (Stoner) enhancement is small, that is $U \rho_{0}<1, \mathcal{S}$ can be enhanced by a factor 10 when the disorder is sufficiently great $\left(k_{F} \lambda \simeq 1\right)$. Fig. 2 shows the same situation in two-dimensions. The insets in the above two figures show that the approximate formulas of Eqs. $(17,18)$ for $\mathcal{S}$ in three and two dimensions are sufficiently accurate except at the largest values of the Stoner factor. Since experiments usually give the Korringa ratio $\mathcal{K}$ directly, we plot it in Figs. 3, 4 as a function of disorder and interaction strength. In all the two-dimensional plots we chose the cutoff $\kappa=1 / \sqrt{20 k_{F} \lambda}$

In Fig. 5, we show a result which is potentially useful for a controlled study in $\mathrm{M}_{3} \mathrm{C}_{60}$ where properties appear to depend rather universally on lattice spacing. For example, in the series $\mathrm{M}=\mathrm{K}_{3-x} \mathrm{Rb}_{x}$ the variations in $T_{c}$, susceptibility [9] and $1 / T_{1}$ [7] have been measured. At present, there seems to be no substantial variation of resistivity with $x$, 10,11 thus we take $k_{F} \lambda$ fixed in the plot which shows the change in $1 / T_{1} T$ relative to that of the uniform susceptibility $\chi$ as the bare density of states $\rho_{0}$ is varied. At the present stage, the uncertainties in the measurements prevent us from making a good inference of the value of the Stoner enhancement or of the bare density of states, but we expect that with increased experimental accuracy, we should be able to exploit the present method to infer such quantities, which are of fundamental interest.

As a matter of fact, Knight shift and NMR relaxation rate data for dilute non-magnetic alloys have been used for many years to study electronic structure in these materials. 12] In view of the disorder enhancement to $1 / T_{1}$ which we have discussed, the systematics of the behavior with impurity concentration should be included in all such analyses. More recently, NMR has been used to study the properties of the rare-earth and actinide heavy electron materials. [13] For example, the superconducting compounds $\mathrm{U}_{1-x} \mathrm{Th}_{x} \mathrm{Be}_{13}$ are of special interest because 
of the dramatic effects caused by the Th doping. There is a large increase in $1 / T_{1}$ just above $T_{c}$ as $x$ goes from 0 to 0.033 [14] which is not well-understood but has been attributed to a density of states effect. Again, it is important to include the effects of disorder on the relaxation rate, as we have discussed.

A system that does show the suggested enhancements in $1 / T_{1} T$, is the well-studied $\left.\mathrm{Si}: \mathrm{P} \| 15,16\right]$, where the observed NMR relaxation rates are approximately three orders of magnitude larger than the clean free electron values. Here there is the added complication that there are two species of relaxing fluids, the dirty electron gas as in the present paper, and the singlet pairs formed by local moments that fluctuate and relax the nuclei in a distinctive fashion as described in [17]. The detailed quantitative breakup between these terms is not easy to do and is not attempted here. The point we wish to make is that the free electron gas component contributes a very large amount (see Figs. 1-2) to the observed $1 / T_{1} T$ when both Stoner enhancement and disorder are present.

\section{ACKNOWLEDGMENTS}

We thank many generous experimental colleagues who have shared their data and wisdom with us: A. Hebard, M. Paalanen, T. Palstra, A. Ramirez, R. Tycko, R. Walstedt, T. Imai. This work was supported in part by NSF grant DMR92-21907 (EA).

[1] J. Korringa, Physica 16, 601 (1950).

[2] D. Pines, Phys. Rev. 92, 626 (1954)

[3] Tôru Moriya, J. Phys. Soc. Japan 18, 516 (1963).

[4] S. Doniach, J. Appl. Physics 39, 483 (1968).

[5] Peter Fulde and Alan Luther, Phys. Rev. 170, 570 (1968).

[6] B. Sriram Shastry, Phys. Rev. Lett. 63, 1288 (1989).

[7] R. Tycko, G. Dabbagh, M. Rosseinsky, D. Murphy, A. Ramirez, and R. Fleming, Phys. Rev. Lett. 68, 1912 (1992), W. Hong, M. Hanson, W. Clark, J. Thomson, R. Whetten, S. Huang, R. Kaner, F. Diedrich, P. Petit, J. Andre and K. Holczer, Europhysics Letts 18, 79 (1992).

[8] T.V. Ramakrishnan and P.A. Lee, Rev. Mod. Phys. 57, 287 (1985).

[9] A. Ramirez, M.J. Rossiensky, D.W. Murphy, and R.C. Haddon, Phys. Rev. Lett. 69, 1687 (1992).

[10] A.F. Hebard, T.M. Palstra, R.C. Haddon, and R.M. Fleming, unpublished.

[11] T.M. Palstra, R.C. Haddon, A.F. Hebard and J. Zaanen, Phys. Rev. Letts, 68, 1054 (1992).

[12] A. Narath, CRC Reviews in Solid State Sciences, March 1972. 
[13] See, for example, the review of K. Asayama et al, J. Magn. Magn. Mat. 76-77, 449 (1988).

[14] D.E. MacLaughlin et al, Phys. Rev. Lett. 53, 1833 (1984).

[15] M. A. Paalanen, A.E. Ruckenstein and G. A. Thomas, Phys. Rev. Lett. 54, 1295 (1985).

[16] H. Alloul and P. Dellouve, Phys. Rev. Lett. 59, 578 (1987).

[17] Zi-Zhao Gan and Patrick A. Lee, Phys. Rev. B 33, 3595 (1986).

FIG. 1. $\mathcal{S}$ versus $X=\rho_{0} U$ in 3-dimensions for $k_{F} \lambda=1,3,5,10, \infty$ from top to bottom. Inset compares the leading approximation (dashed lines) Eq. (18) with the exact numerics (solid lines) for $k_{F} \lambda=1,10$

FIG. 2. $\mathcal{S}$ versus $X=\rho_{0} U$ in 2-dimensions for $k_{F} \lambda=1,3, \infty$ from top to bottom. Inset compares the leading approximation (dashed lines) Eq. (17) with the exact numerics (solid lines) for $k_{F} \lambda=1,10$

FIG. 3. The Korringa ratio $\mathcal{K}$ versus $X=\rho_{0} U$ in 3- dimensions for $k_{F} \lambda=1,3,5,10, \infty$ from top to bottom.

FIG. 4. The Korringa ratio $\mathcal{K}$ versus $X=\rho_{0} U$ in 2- dimensions for $k_{F} \lambda=1,3,5,10, \infty$ from top to bottom.

FIG. $5 . \quad$ The logarithmic derivative $\Gamma=d \ln \left[\left(\rho_{0} U\right)^{2} \mathcal{S}\left(\rho_{0} U, k_{F} \lambda\right)\right] / d \ln \left[\rho_{0} U /\left(1-\rho_{0} U\right)\right]$ versus $X=\rho_{0} U$ in $3 D$. This corresponds to a variation of the bare density of states for a fixed $U$ and the resulting change in $1 / T_{1} T$ relative to that in the uniform susceptibilty $\chi$ on a logarithmic scale, i.e. $d \ln \left(1 / T_{1} T\right) / d \ln \chi$. Upper (lower) curve is for $k_{F} \lambda=1(\infty)$ 